\title{
Dynamics of colistin and tobramycin resistance among Enterobacter cloacae during prolonged use of selective decontamination of the digestive tract
}

\author{
M. J. D. Dautzenberg ${ }^{1,2,3^{*}}$, J. R. Bayjanov' ${ }^{1}$, M. A. Leverstein-van Hall ${ }^{4,5}$, A. E. Muller ${ }^{4}$, L. B. S. Gelinck ${ }^{6}$, C. L. Jansen ${ }^{4}$,
} E. M. S. Leyten ${ }^{6}$, T. Ruys ${ }^{7}$, J. Scharringa ${ }^{1}$, R. E. van der Starre ${ }^{4}$, A. C. Fluit ${ }^{1}$ and M. J. M. Bonten ${ }^{1,2}$

\begin{abstract}
Background: A high prevalence of colistin resistance among E. cloacae isolates in two intensive care units (ICU) (of 16 and 6 beds) using selective digestive decontamination (SDD) since 1990 instigated a retrospective and prospective investigation to quantify the role of clonal transmission. SDD is topical application of colistin and tobramycin and systemic use of cefotaxime during the first days of ICU-admission.

Methods: Multi-resistant E. cloacae (MREb) was defined as ESBL production and/or tobramycin non-susceptibility and/or colistin non-susceptibility. Incidence of acquisition and prevalence of carriage with MREb was determined from microbiological culture results.

Results: Colistin-resistant E. cloacae was first detected in November 2009 and carriage was demonstrated in 141 patients until October 2014. Mean incidence of MREb acquisition was 4.61 and 1.86 per 1000 days at risk in ICUs 1 and 2, respectively, and the mean monthly prevalence of MREb in both ICUs was 7.0 and 3.1\%, respectively, without a discernible trend in time. Conversion rates from carriage of colistin-susceptible to resistant E. cloacae were 0.20 and 0.13 per 1000 patient days, respectively. Whole genome sequencing of 149 isolates revealed eight clusters, with the number of SNPs of the largest two clusters ranging between 0 and 116 for cluster 1 ( $n=49$ isolates), and 0 and 27 for cluster 2 ( $n=36$ isolates), among isolates derived between 2009 and 2014.
\end{abstract}

Conclusions: This study demonstrates a stable low-level endemicity of MREb in two Dutch ICUs with prolonged use of SDD, which was characterized by the persistent presence of two clusters, suggesting incidental clonal transmission.

Keywords: Colistin resistance, Tobramycin resistance, Enterobacter cloacae, Intensive care unit, Selective digestive tract decontamination

\section{Background}

Enterobacter cloacae are commensal bacteria of the human gut that can inactivate third-generation cephalosporins through overproduction of chromosomally encoded AmpC beta-lactamases, especially during treatment with these antibiotics. Therefore, recommended treatment of infections with these bacteria

\footnotetext{
* Correspondence: mirjam.dautzenberg@radboudumc.nl

'Department of Medical Microbiology, University Medical Center Utrecht, Utrecht, the Netherlands

${ }^{2}$ Julius Center for Health Sciences and Primary Care, University Medical Center Utrecht, Utrecht, the Netherlands

Full list of author information is available at the end of the article
}

includes trimethoprim/sulfamethoxazole, fluoroquinolones or carbapenem antibiotics.

The E. cloacae complex comprises five species: $E$. cloacae, Enterobacter asburiae, Enterobacter hormaechei, Enterobacter kobei, and Enterobacter ludwigii. E. cloacae and $E$. hormaechei are the most frequently isolated human isolates [1]. Classification beyond genus level is difficult, and therefore often not performed. Aminoglycoside resistance in Enterobacter species is usually attributable to aminoglycoside-modifying enzymes that are often plasmid-encoded but can also be associated with transposable elements [1]. Colistin resistance is mostly caused by

(c) The Author(s). 2018 Open Access This article is distributed under the terms of the Creative Commons Attribution 4.0 International License (http://creativecommons.org/licenses/by/4.0/), which permits unrestricted use, distribution, and reproduction in any medium, provided you give appropriate credit to the original author(s) and the source, provide a link to the Creative Commons license, and indicate if changes were made. The Creative Commons Public Domain Dedication waiver (http://creativecommons.org/publicdomain/zero/1.0/) applies to the data made available in this article, unless otherwise stated. 
chromosomal mutations, most likely inducing changes in the negatively charged surface lipopolysaccharides [2]. Recently, plasmid-mediated resistance encoded by different $m c r$ genes has been described [3].

Antibiotics may create a selective pressure leading to detectable carriage with Enterobacter species, especially in hospitalized patients (endogenous selection). Although such new detections are often considered acquisition, they do not represent true acquisition events. An increased incidence of healthcare-associated infections or carriage with $E$. cloacae can, therefore, result from endogenous selection, but also from clonal transmission, either or not facilitated by selective antibiotic pressure. Bacterial typing is required to disentangle the relative importance of both acquisition routes.

Selective decontamination of the digestive tract (SDD) is an infection prevention measure consisting of mouthpaste and intragastric suspension containing tobramycin, colistin and amphotericin B, and intravenous cefotaxime during the first days of ICU admission. Selective oropharyngeal decontamination (SOD) only includes the mouthpaste, with the same topical antibiotics. Based on studies in which SDD and SOD were associated with lower ICU mortality, SOD or SDD became standard-ofcare in Dutch ICUs [4]. The effect of SDD and SOD on the emergence of colistin resistance among Gram negative bacteria is unknown. In longitudinal studies in Dutch ICUs using SDD this risk was low [5], but outbreaks with colistin-resistant bacteria during SDD have been reported as well [6].

A high incidence of carriage and infection with colistinresistant $E$. cloacae complex isolates in patients admitted in the intensive care unit (ICU) in a Dutch hospital was noted in June 2014. It was hypothesized that the use of SDD in this ICU since 1990 had contributed to this high incidence, either due to repeated events of endogenous selection or due to incidental introduction of colistin-nonsusceptible strains followed by clonal transmission. A detailed retrospective and prospective investigation was performed to investigate the epidemiology of antibioticresistant $E$. cloacae complex isolates.

\section{Methods}

\section{Setting}

The hospital is a 540-bed secondary care hospital with two ICUs (16 and 6 beds) on physically separated locations. There is intensive exchange of employees and patients between both ICUs. SDD consisting of mouthpaste (500 mg 4 times daily) and intragastric suspension (8 $\mathrm{ml} 4$ times daily) (tobramycin, colistin, and amphotericin B) and intravenous cefotaxime $1000 \mathrm{mg} 4$ times daily for the first 3 days of ICU admission, was used routinely for patients with an expected length of invasive ventilation of $>24 \mathrm{~h}$ and patients receiving enteral tube feeding for an expected length of ICU stay of $>48 \mathrm{~h}$, since 1990. As part of the SDD strategy, screening was performed on admission and twice weekly using throat and rectum swabs. This standard of care was interrupted only between 2004 and 2006 as part of a multicentre trial evaluating the effects of SDD, in which SOD was used for 6 months and neither SDD nor SOD were used for another 6 months [4].

\section{Data collection}

Demographic data, patient location data and data on antibiotic use were extracted from the electronic hospital information system. Data was collected from 1 May 2005 to 1 November 2014. Data on antibiotic prescriptions are available from 1 November 2005, and data on patient location from 1 January 2007. Microbiological data was extracted from the electronic laboratory system, and data were available from 1 May 2005 onwards. Colistin susceptibility was documented since November 2009 and presence of ESBL since January 2011.

\section{Microbiological methods}

Species identification and susceptibility testing of clinical isolates was performed using Vitek 2 (bioMérieux, Marcy-l'Étoile, France). Isolates with elevated MIC for meropenem and/or imipenem were sent to a reference laboratory to test for presence of carbapenemases. Pulsed-field gel electrophoresis (PFGE) had been performed on 96 Enterobacter isolates from 62 patients and 8 environmental samples as screening (data not shown).

For isolates selected for whole-genome sequencing (WGS, as described below) species identification was performed by MALDI-TOF (Microflex, Bruker, Germany), antibiotic susceptibility testing was performed by Vitek 2, colistin resistance was additionally determined by Etest (bioMérieux, Marcy-l'Étoile, France), and presence of ESBL by cefepime-clavulanate ESBL Etest (bioMérieux, Marcy-l'Étoile, France), both on Mueller Hinton 2 agar.

\section{Definitions}

Multi-resistant Enterobacter species (MREb) was defined as Enterobacter isolates with ESBL production and/or tobramycin non-susceptible (MIC $>4 \mathrm{mg} / \mathrm{L}$; EUCAST 2013 [7]) and/or colistin resistant (MIC> $2 \mathrm{mg} / \mathrm{L}$; EUCAST 2013). Furthermore, Enterobacter isolates were classified on resistance profile as I) tobramycin nonsusceptible Enterobacter spp., II) colistin resistant Enterobacter spp., or III) ESBL producing Enterobacter spp.

Selection of isolates for whole genome sequencing (WGS) First, from each patient from whom Enterobacter spp. isolates had been stored, isolates in each of the following groups were selected for WGS a) isolates for which PFGE had been performed before, b) blood culture 
isolates, c) cerebrospinal fluid (CSF) isolates, d) colistinresistant isolates, e) isolates from ICU. When multiple isolates for one individual patient were available in one of the groups, the final selection per patient was based on 1) location (ICU, ward, outpatient), 2) highest MIC for colistin, tobramycin and ESBL positivity, 3) the most invasive isolate (culture site: blood, CSF, broncho-alveolar lavage, fluid/punctate, wound, urine, sputum, catheter tip, throat, rectum, nose, faeces), 4) culture date (first).

From one patient both colistin-susceptible $(n=2)$ and resistant $E$. cloacae complex isolates $(n=1)$ were selected for WGS. For one patient isolates collected 4 years apart were selected (colistin susceptible, tobramycin susceptible).

As control isolates, which were considered not be linked to the local hospital epidemiology, we included 23 Enterobacter isolates; 17 cultured between 2002 and 2014 in another Dutch hospital in the same region, one from Curacao, an island in the Caribbean Sea, and six unrelated isolates.

To test whether colistin resistance had been induced in susceptible isolates that were prevalent in the geographic area, 15 susceptible isolates were also included. These included seven isolates from patients admitted in the same period, six isolates from general practice patients and two isolates from a different hospital in the same city. None of these patients had documented carriage with MREb.

\section{Whole genome sequencing}

Isolates were cultured overnight at $37{ }^{\circ} \mathrm{C}$ on blood agar plates. Subsequently one colony was transferred to Lysogeny Broth and incubated overnight at $37^{\circ} \mathrm{C}$. From this culture DNA was isolated using the Ultra Clean Microbial DNA isolation kit (Mo Bio Laboratories, Inc., Carlsbad, CA). Library preparation used the Illumina Nextera XT DNA Sample Preparation kit and sequencing was performed on an Illumina NextSeq with the mid-output $2 \times 150$ bp kit using paired-ends (Illumina, San Diego, CA).

\section{Comparative genomics analysis}

In total, genomic DNA of 172 Enterobacter cloacae complex isolates were sequenced. Sequence reads were first quality-filtered using seqtk with option "trimfq -q 0.01" (version 1.0-r31) [8] and then assembled using SPAdes (version 3.7.0) [9]. Genomes with lengths ranging between $3 \mathrm{Mb}$ and $8 \mathrm{Mb}$ were selected for further analysis, which resulted in a total of 150 genomes. Assembled contigs were annotated using prokka (version 1.12-beta) [10]. Proteins among these strains were first aligned against each other using BLAST+ (version 2.2.31) [11], where length of aligned part must be longer than the half length of both query and reference sequences. Resulting alignment file was used as an input to
orthAgogue (version 1.0.3) [12] to find orthologous proteins and orthologous proteins were clustered using $\mathrm{mcl}$ (version 12-135) [13]. Genes that are present in all strains (core genes) were concatenated to create a core genome. Next, recombination regions in the core genome were identified using gubbins (version 1.4.9) [14]. Subsequently, all gene sequences in identified recombination regions were discarded and final recombinationfiltered core genomes were used to build a phylogenetic tree using FastTree2 (version 2.1.8) with the GTR model and with 100 bootstrap samples [15].

\section{Statistical analyses}

Incidence of acquisition in ICU was calculated per year as number of patients with a positive culture according to one of the classifications, per 1000 patient days at risk. Acquisition was defined as growth of Enterobacter spp. from a microbiological culture on ICU-day 3 or thereafter, with a preceding negative culture. Admissions $<48 \mathrm{~h}$ were not included in this analysis as these were not considered at risk for acquisition according to this definition. Patient days at risk are the sum of all uncolonized days in ICU of all patients until ICU-discharge or until the day of the first culture with Enterobacter spp. Prevalence is calculated as the percentage of colonized patient days divided by the total number of patient days. Sensitivity and specificity of determination of colistin resistance using Vitek were calculated with Etest as reference standard. Data analysis was performed using SPSS version 22 and $\mathrm{R}$ version 3.1.2.

\section{Results}

\section{Microbiology results}

From 1 May 2005 to 1 November 2014 Enterobacter spp. were detected in 2448 patients in both ICUs, including 1373 MREb isolates from 373 patients. These isolates were derived from rectal/fecal samples $(n=105 ; 28.2 \%)$, wounds $(n=71 ; 19.0 \%)$, sputum $(n=66 ; 17.7 \%)$, urine $(n=53$; $14.2 \%)$, throat/nose $(n=48 ; 12.9 \%)$, catheter tips $(n=8$; $2.1 \%)$, broncho-alveolar lavage fluid $(n=7 ; 1.9 \%)$, blood $(n=6 ; 1.6 \%)$ and other material $(n=9 ; 2.4 \%)$. Among the MREb isolates proportions of nonsusceptibility were $68.8 \%$ for colistin (141 of 205 tested), $89.3 \%$ for tobramycin (333 of 373 tested) and $71.2 \%$ had ESBL production (111 of 156 tested) (Table 1). Of the MREb isolates, $82.3 \%$ was susceptible to either ciprofloxacin or trimethoprim/sulfamethoxazole, $99.1 \%$ to ciprofloxacin, trimethoprim/sulfamethoxazole or meropenem, and 99.5\% to ciprofloxacin, trimethoprim/sulfamethoxazole, meropenem or colistin. Twenty-two of 373 patients with MREb had isolates with elevated MICs for imipenem and/ or meropenem. PCR testing for carbapenemase-producing genes was performed for isolates of 12 patients and New Delhi metallo-beta-lactamase (NDM) was detected in one 
Table 1 Antibiotic resistance in patients with resistant E. cloacae and susceptible E. cloacae

\begin{tabular}{|c|c|c|c|c|c|}
\hline \multirow[t]{2}{*}{ Antibiotic (combination) } & \multicolumn{2}{|c|}{$\begin{array}{l}\text { Susceptible E. cloacae } \\
\text { ( } n=2075 \text { unique patients, } 3030 \text { isolates) }\end{array}$} & \multicolumn{2}{|c|}{$\begin{array}{l}\text { Multi-resistant } E \text {. cloacae } \\
(n=373 \text { patients, } 1373 \text { isolates) }\end{array}$} & \multirow{2}{*}{$\begin{array}{l}\text { Selected for WGS } \\
\text { ( } n=112 \text { isolates) } \\
\% \text { non- susceptible / } \\
\text { positive }^{\text {a }}\end{array}$} \\
\hline & $\begin{array}{l}\text { Patients (n) } \\
\text { tested }\end{array}$ & $\begin{array}{l}\% \text { non-susceptible / } \\
\text { positive }^{a}\end{array}$ & $\begin{array}{l}\text { Patients (n) } \\
\text { tested }\end{array}$ & $\begin{array}{l}\% \text { non- susceptible / } \\
\text { positive }^{\mathrm{a}}\end{array}$ & \\
\hline ciprofloxacin & 2075 & 1.3 & 373 & 42.9 & 28.6 \\
\hline colistin & 1122 & 0 & 205 & 68.8 & $78.4^{b}$ \\
\hline gentamicin & 2075 & 0.2 & 373 & 82.8 & 86.6 \\
\hline imipenem & 2070 & 0.2 & 373 & 5.6 & 6.3 \\
\hline meropenem & 2073 & 0.1 & 373 & 4.8 & 6.3 \\
\hline tobramycin & 2075 & 0 & 373 & 89.3 & 87.5 \\
\hline trimethoprim/ sulfamethoxazole & 2075 & 5.5 & 373 & 34.6 & 86.6 \\
\hline ESBL production & 873 & 0 & 156 & 71.2 & 72.3 \\
\hline CIP or SXT & 2075 & 0.6 & 373 & 17.7 & 5.4 \\
\hline CIP or SXT or MEM & 2064 & 0 & 349 & 0.9 & 0 \\
\hline CIP or SXT or MEM or CST & 1118 & 0 & 187 & 0.5 & 0 \\
\hline
\end{tabular}

CIP ciprofloxacin, CST colistin, ESBL extended-spectrum beta-lactamase, MEM meropenem, $n$ number, SXT trimethoprim/ sulfamethoxazole, WGS whole-genome sequencing a\% non-susceptible for antibiotics or \% ESBL positive

${ }^{\mathrm{b}} 111 / 112$ isolates tested

Number of resistant $E$. cloacae isolates tested: ciprofloxacin: $n=1373$, colistin: $n=784$, gentamicin: $n=1373$, imipenem: $n=1373$, meropenem: $n=1371$, tobramycin: $n=1373$, trimethoprim/ sulfamethoxazole: $n=1372$. Number of susceptible E. cloacae isolates tested: ciprofloxacin: $n=3030$, colistin: $n=1613$, gentamicin: $n=3030$, imipenem: $n=3024$, meropenem: $n=3028$, tobramycin: $n=3030$, trimethoprim/ sulfamethoxazole: $n=3030$

isolate. This patient had been hospitalized for 1 day in 2011, after a recent return from Mecca, Saudi Arabia.

From 1 January 2005 onwards there were 97 E. cloacae bacteremia episodes in 96 patients, and 13 isolates (13.5\%) were non-susceptible to tobramycin. Since 2009, though, a tobramycin and colistin non-susceptible isolate was obtained from only one of 42 patients (2.4\%) with E. cloacae bacteremia.

Comparison of colistin susceptibility testing by Vitek and Etest of 100 isolates (86 being resistant) yielded sensitivity and specificity of $100 \%$ (Additional file 1 : Table S1).

\section{Incidence and prevalence of MREb}

There were (together in both ICUs) 12,680 ICU admissions of 10,741 patients between 1 January 2007 and 31
October 2014; 3506 admissions (3210 patients) had an ICU-stay $>=48 \mathrm{~h}$. On admission prevalence of MREb was $1.7 \%$ (61/3506; 61 admissions of 49 patients), and 154 patients acquired MREb during ICU-stay (Table 2). In 73 patients carriage or infection was detected after ICU discharge. Of the 373 patients with MREb, 146 could not be related to stay in either of the two ICUs. For 102 of these 146 patients data collection was incomplete, as ICU admission data were available from 2007 onwards.

Incidence rates of MREb acquisition per 3 months from 2007 to 2014 are shown in Figs. 1 and 2. MREb acquisition rates ranged from 4 to 7 per 1000 days at risk, with no discernable trend in time. Similar ranges of acquisition were observed for the three individual resistance markers.

Table 2 Incidence of resistant E. cloacae in ICU 1 and 2

\begin{tabular}{|c|c|c|c|c|c|c|}
\hline & \multirow[t]{2}{*}{ Selection } & \multicolumn{2}{|l|}{ ICU 1} & \multicolumn{2}{|l|}{ ICU 2} & \multirow[t]{2}{*}{ Period } \\
\hline & & All admissions & $\overline{\text { Admissions }>48 \mathrm{~h}}$ & All admissions & $\overline{\text { Admissions }>48 \mathrm{~h}}$ & \\
\hline$\overline{\text { Admissions (n) }}$ & \multirow[t]{2}{*}{ All } & 9418 & 2731 & 3624 & 884 & 1 Jan 2007-1 Nov 2014 \\
\hline Patients (n) & & 8073 & 2301 & 3257 & 836 & 1 Jan 2007-1 Nov 2014 \\
\hline On admission & \multirow[t]{2}{*}{ MREb } & $58^{\mathrm{a}}$ & & $25^{\mathrm{a}}$ & & 1 Jan 2007-1 Nov 2014 \\
\hline Acquisitions (n) & & 134 & & 20 & & 1 Jan 2007-1 Nov 2014 \\
\hline \multirow{4}{*}{$\begin{array}{l}\text { Mean incidence } \\
\text { (acquisitions/DAR) }\end{array}$} & MREb & $4.60(69 / 14977)$ & & $1.85(6 / 3248)$ & & 1 Jan 2011-1 Nov 2014 \\
\hline & Tobramycin I or R & $4.95(130 / 26265)$ & & $2.74(20 / 7312)$ & & 1 Jan 2007-1 Nov 2014 \\
\hline & Colistin R & $5.02(81 / 16139)$ & & $2.28(10 / 4386)$ & & 1 Jan 2010-1 Nov 2014 \\
\hline & ESBL production & $4.64(58 / 12489)$ & & $1.88(6 / 3200)$ & & 1 Jan 2011-1 Nov 2014 \\
\hline
\end{tabular}

$D A R$ days at risk, ESBL extended-spectrum beta-lactamase, I intermediate, ICU intensive care unit, MREb extended-spectrum beta-lactamase production and/or tobramycin non-susceptible and/or colistin-resistant, $n$ number, $R$ resistant. Colistin susceptibility was documented since December 2009 and presence of ESBL since January 2011

${ }^{a}$ These numbers include patients transmitted between the two ICUs. In total in 61 ICU admissions MREb was detected before or on admission 

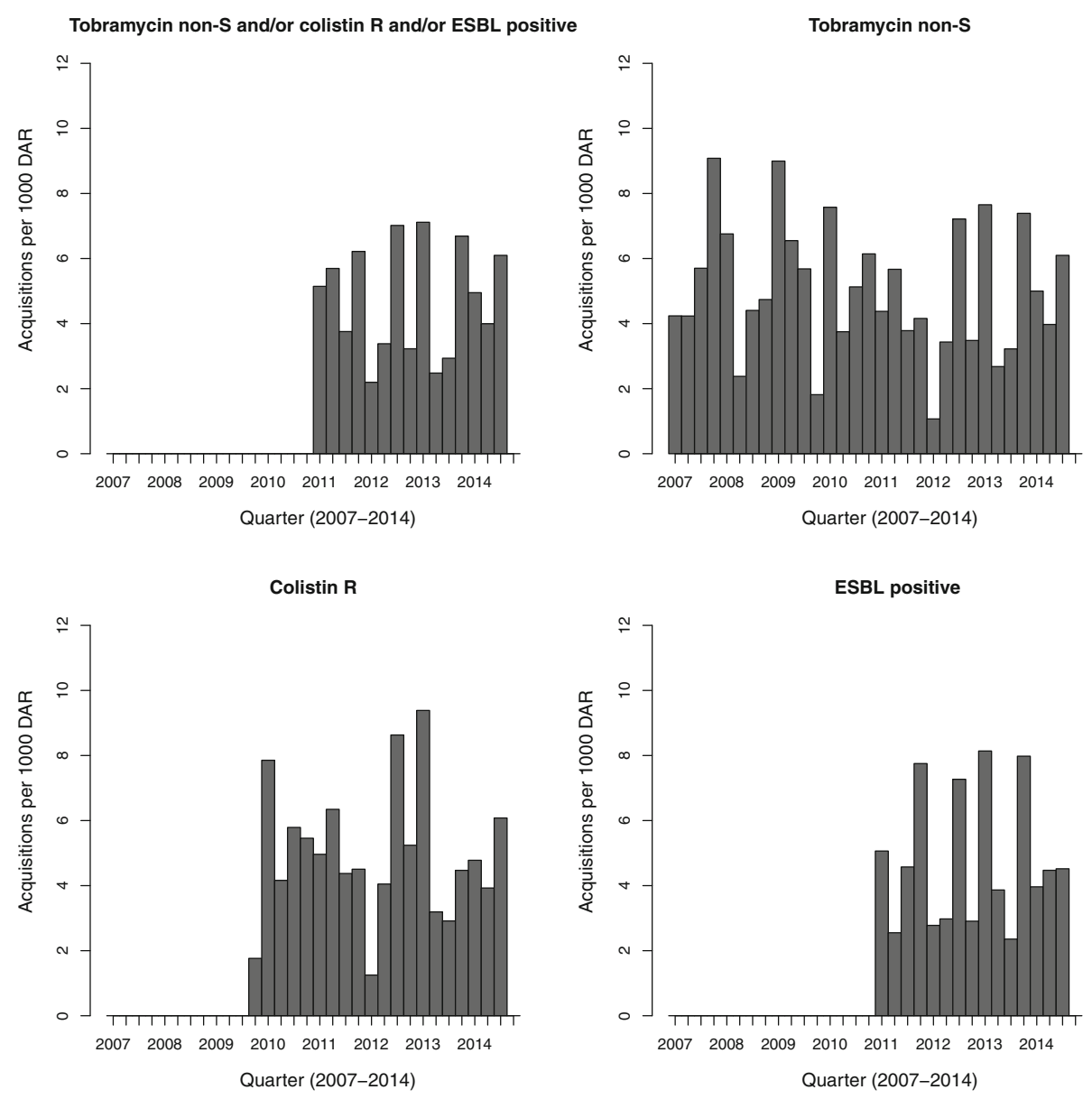

Fig. 1 Incidence of resistant E. cloacae on ICU 1. ESBL: extended-spectrum beta-lactamase, non-S: non-susceptible, R: resistant

MREb was regularly present in ICU 1 , with at least one patient carrying MREb being present in $53.8 \%$ of all days, and a monthly mean prevalence of $7.0 \%$ (19.6 per 282 patient days). MREb was incidentally present in ICU 2 , with $8.9 \%$ of days at least one patient carrying MREb admitted, and monthly mean prevalence of $3.1 \%$ (2.5 per 80 patient days; Fig. 3). There was no discernable trend in prevalence in time, with more variation in prevalence in ICU 2 because of the lower number of patients admitted at each moment.

From 2007 onwards, up to 81 patients had not been admitted to ICU before their first positive culture with resistant E. cloacae.

\section{Acquisition of colistin resistance}

A change in colistin susceptibility (from susceptible to resistant) of $E$. cloacae detected in screening cultures was observed in three patients with rectal colonization and in two other patients with respiratory colonization, corresponding to conversion rates of 0.20 and 0.13 conversions per 1000 patient days of $E$. cloacae carriage. All five patients received SDD.

\section{Whole genome sequencing}

Of 362 stored E. cloacae isolates, 112 isolates from 96 patients were selected for WGS. Susceptibility profiles of these isolates are shown in Table 1. The phylogenetic tree was built on 112 MREb isolates, 15 susceptible isolates, and 23 control isolates (Fig. 4). WGS revealed eight clusters, of $49,38,3,5,3,2,2$ and 19 isolates respectively, with the number of SNPs of the largest two clusters ranging between 0 and 116 for cluster A $(n=49$ isolates), and 0 and 27 for cluster $\mathrm{B}$ ( $n=38$ isolates). The oldest cluster A isolate was identified in June 2009 and the youngest in May 2014. Cluster B isolates were derived between December 2009 and August 2014.

There was a high level of concordance in antibiotic susceptibility within clusters, except for ESBLproduction (Table 3 ). In clusters $\mathrm{A}$ and $\mathrm{B}$, the majority of isolates were non-susceptible to colistin (46 of 47 isolates tested and 37 of 38 isolates tested), and nonsusceptible to tobramycin (46 of 47 isolates tested and 38 of 38 isolates tested). Seventy-two were ESBLproducers (41 of 45 isolates tested and 31 of 33 isolates tested). All cluster $\mathrm{C}$ to $\mathrm{G}$ isolates are colistin and 

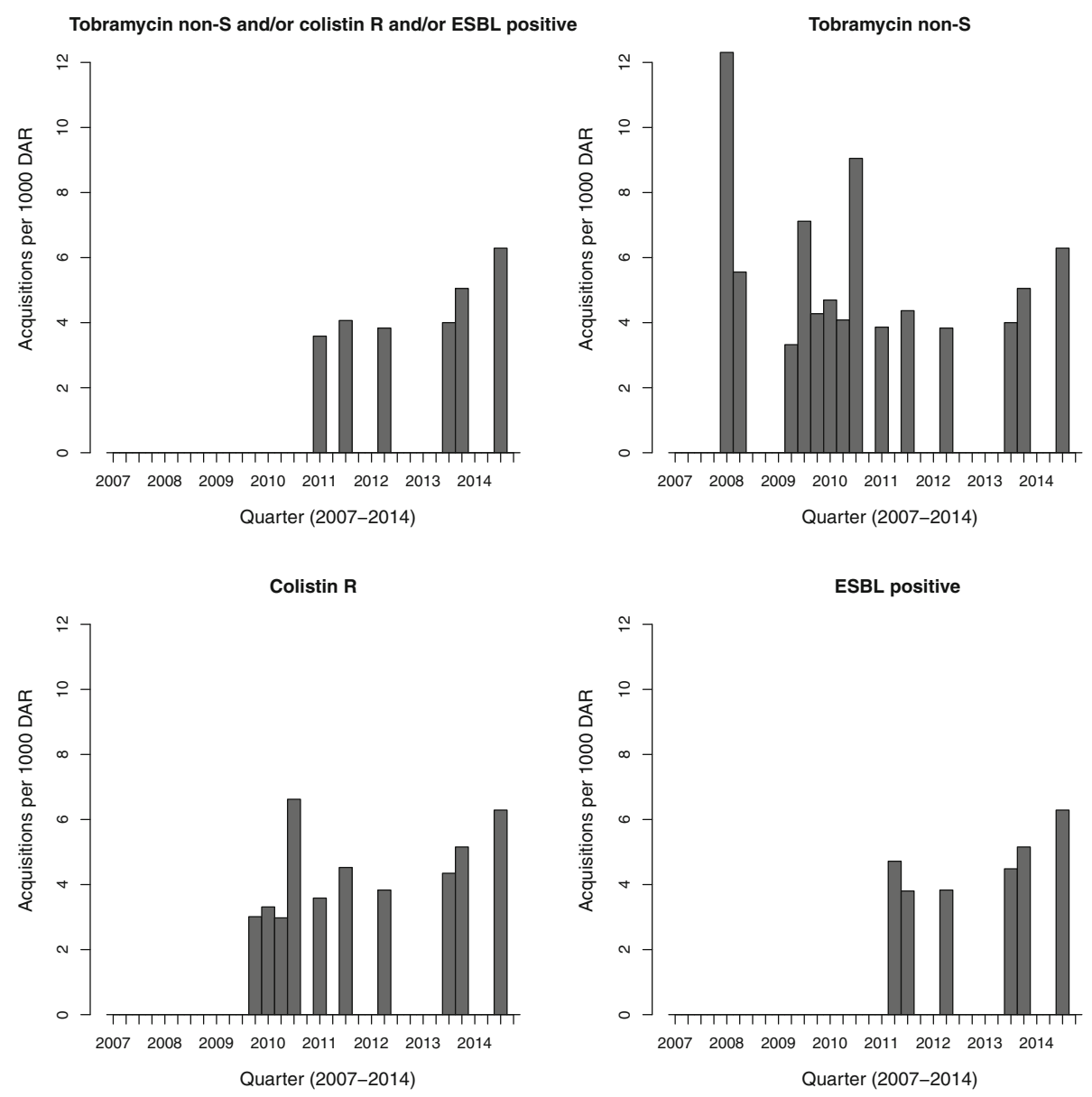

Fig. 2 Incidence of resistant E. cloacae on ICU 2. ESBL: extended-spectrum beta-lactamase, non-S: non-susceptible, R: resistant

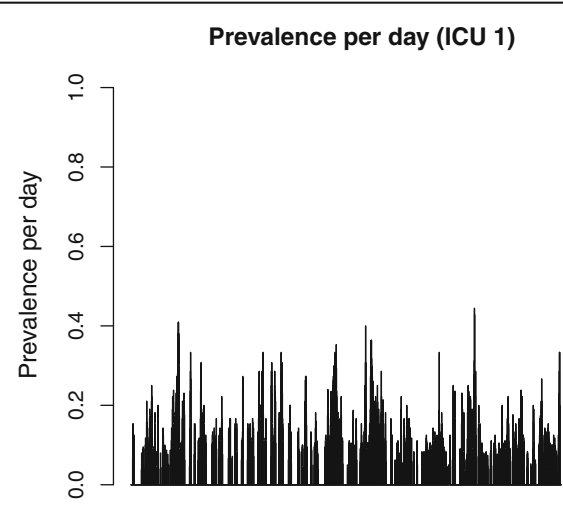

Days (2007-2014)
Prevalence per day (ICU 2)

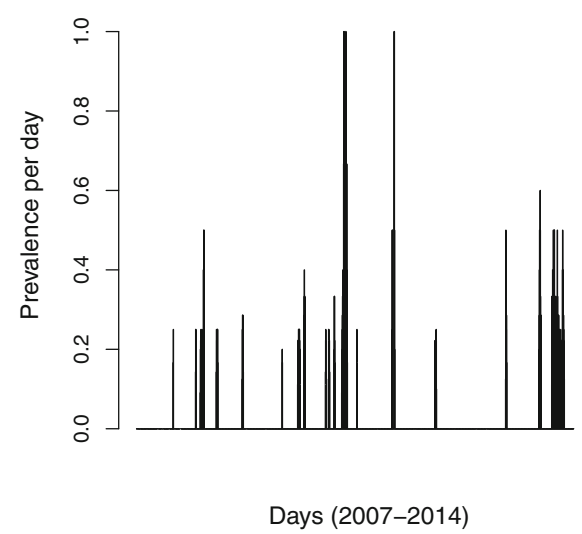

Fig. 3 Daily prevalence of ESBL producing and/or tobramycin non-susceptible and/or colistin-resistant E. cloacae, in ICU (ICU 1 and ICU 2) 


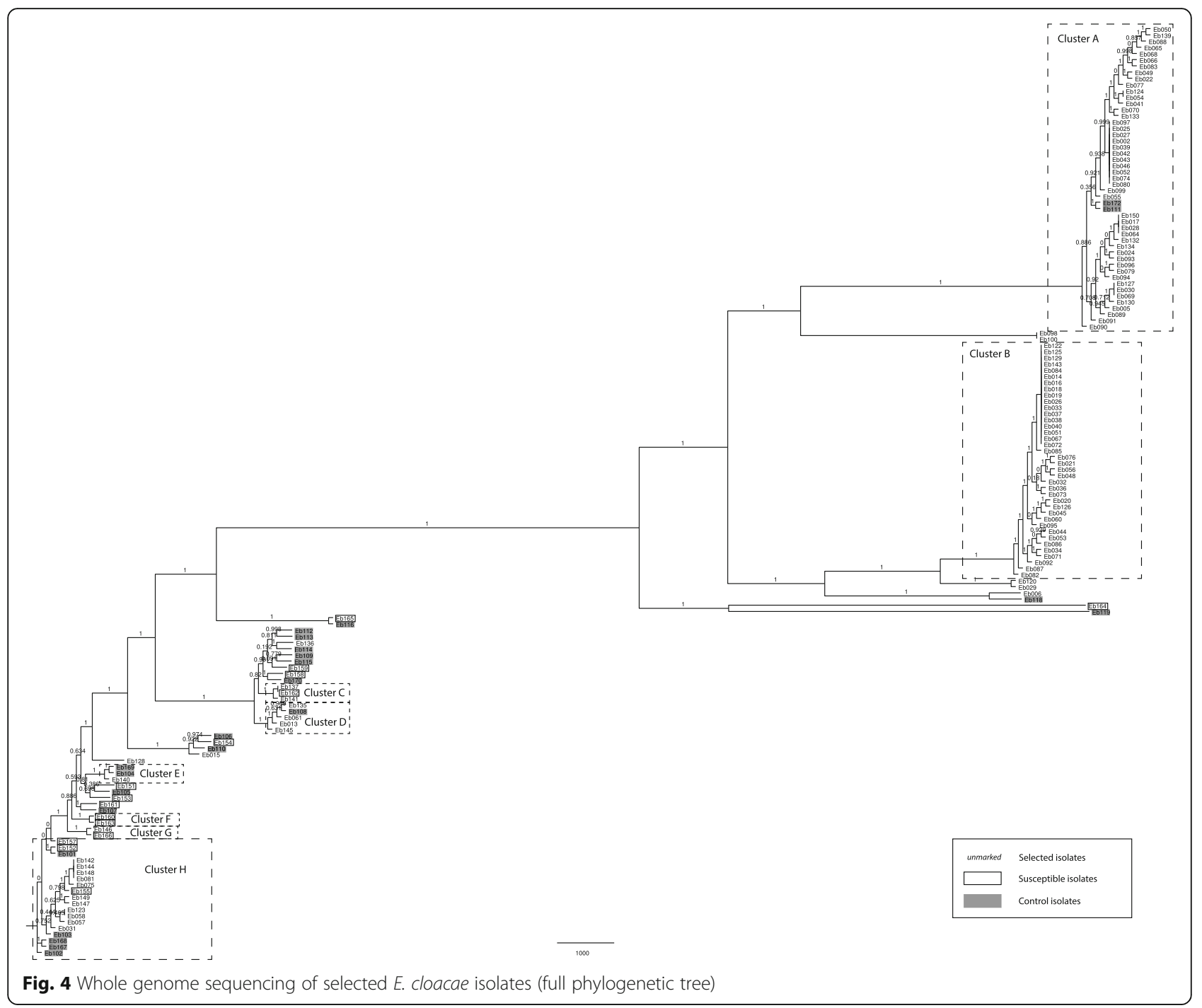

tobramycine-susceptible. All but one cluster $\mathrm{H}$ isolates are colistin susceptible and tobramycine resistant. This isolate is the only isolate outside of clusters A and B that is both tobramycin and colistin non-susceptible. In the one patient with three isolates tested, two non-MREb bacteremia isolates (Eb098 and Eb100) were clearly distinct from the MREb isolate (Eb099) in cluster A.

\section{Discussion}

This study demonstrates a persistently stable low-level endemicity of Enterobacter species resistant to colistin and/or tobramycin in a Dutch ICU that used SDD since 1999. The incidence of acquisition of resistant E. cloacae was 4.61 and 1.86 per 1000 admission days for ICU 1 and 2 respectively, and the mean prevalence was 7 and $3 \%$, respectively. Although $69 \%$ of the resistant isolates were resistant to colistin, susceptibility to either one of the preferred antibiotics for infections caused by Enterobacter species, such as co-trimoxazole, ciprofloxacin or meropenem, was 99\%. Carbapenemase production was demonstrated in one resistant E. cloacae isolate, which appeared to be introduced from abroad. Therefore, resistance in these Enterobacter species did not reduce antibiotic treatment options. WGS revealed two large clusters spanning a prolonged time period, strongly suggesting incidental clonal transmission, sufficient to maintain low-level endemicity, which probably is higher than in most other Dutch ICUs.

Evaluation of long-term trends in antibiotic resistance in ICU isolates in June 2014 led to the recognition of the presence of colistin-resistant Enterobacter species, and initiated the formation of an outbreak management team, including medical microbiologists, infection prevention specialists, intensivists, ICU nurses, infectiologists, and facilitary services. A variety of measures were taken including reinforcement of existing infection prevention measures, including hand hygiene precautions and isolation protocols, replacement of dated equipment, 
Table 3 Characteristics of selected E. cloacae isolates

\begin{tabular}{|c|c|c|c|c|c|c|}
\hline \multirow[t]{2}{*}{ Cluster } & \multirow{2}{*}{$\begin{array}{l}\text { Number } \\
\text { of isolates }\end{array}$} & \multirow{2}{*}{$\begin{array}{l}\text { Location of patients located } \\
\text { in hospital } 1 \text { or } 2 \text { at time of culture }\end{array}$} & \multirow[t]{2}{*}{ Period of detection } & \multicolumn{3}{|c|}{ Resistance ( $\mathrm{n} / \mathrm{n}$ tested) } \\
\hline & & & & Colistin & Tobramycin & ESBL \\
\hline A & 49 & $\begin{array}{l}\text { Intensive care unit } \\
\text { (34 patients ICU 1;5 patients ICU 2) }\end{array}$ & June 2009 - May 2014 & non-S (46/47) & non-S (46/47) & positive $(41 / 45)$ \\
\hline B & 38 & $\begin{array}{l}\text { Intensive care unit } \\
\text { (29 patients ICU } 1 ; 5 \text { patients ICU 2) }\end{array}$ & December 2009 - August 2014 & non-S (37/38) & non-S (38/38) & positive $(31 / 33)$ \\
\hline C & 3 & $\begin{array}{l}\text { Ward } \\
\text { (2 patients hospital 1; 1 patient hospital 2) }\end{array}$ & June 2013 - February 2014 & $S(3 / 3)$ & $S(3 / 3)$ & negative (2/3) \\
\hline D & 5 & $\begin{array}{l}\text { Intensive care unit } \\
\text { (3 patients ICU 1) }\end{array}$ & September 2009 - May 2012 & $S(4 / 4)$ & $S(4 / 4)$ & positive (4/4) \\
\hline $\mathrm{E}$ & 3 & $\begin{array}{l}\text { Ward } \\
\text { (1 patient hospital 1) }\end{array}$ & January 2014 - January 2014 & $S(1 / 1)$ & $\mathrm{S}(1 / 1)$ & positive (1/1) \\
\hline $\mathrm{F}$ & 2 & $\begin{array}{l}\text { Ward/outpatient } \\
(2 \text { patients hospital 1) }\end{array}$ & July 2013 - August 2014 & $S(2 / 2)$ & $S(2 / 2)$ & negative (2/2) \\
\hline G & 2 & $\begin{array}{l}\text { Ward } \\
\text { (1 patient hospital 1) }\end{array}$ & February 2012 - April 2012 & $S(2 / 2)$ & $S(2 / 2)$ & positive $(1 / 2)$ \\
\hline $\mathrm{H}$ & 19 & $\begin{array}{l}\text { Intensive care unit ( } 6 \text { patients hospital } 1) \text {, } \\
\text { ward ( } 5 \text { patients hospital } 1)\end{array}$ & April 2010 - July 2014 & $S(13 / 14)$ & non-S (13/14) & positive $(7 / 14)$ \\
\hline
\end{tabular}

ESBL extended-spectrum beta-lactamase, ICU intensive care unit, $n$ number, non- $S$ non-susceptible, $S$ susceptible

daily infection prevention audits and renovation of some spaces. On 1 September 2014 SDD was replaced by SOD.

The investigation described here started in January 2015 (after all measures had been implemented). The focus of investigation was on the ICU where screening cultures had been obtained routinely as part of SDD. As such, the likelihood of detecting such isolates outside the ICUs is lower. Yet, from 2007 onwards, up to 81 patients had not been admitted to ICU before their first positive culture with resistant $E$. cloacae, suggesting that transmission was not limited to the ICUs.

In this study, colistin susceptibility testing as performed by Vitek appeared adequate in distinguishing resistant from susceptible isolates using Etest as a comparator. It should be noted that gradient tests have recently been tested to generally underestimate MICs, resulting in false susceptible results [16], thus misclassification in our study cannot be ruled out. Yet, there were only a limited number of isolates with an MIC in the intermediate range.

The possible effects of colistin use in SDD could not be disentangled, as SDD was administered to all eligible patients admitted to the ICU, precluding a comparison with control patients. The conversion rate from carriage of colistin-susceptible to resistant $E$. cloacae ( 0.20 and 0.13 conversions per 1000 patient days) is lower than previously reported in ICUs using SDD [5], even though we might have overestimated true conversion in these patients as susceptible isolates of these patients were not routinely stored. Previously, estimated conversion rates for colistin in Enterobacter spp., Escherichia coli or Klebsiella spp. during SDD in rectal isolates were 1.0 and 0.7 per 1000 patient days in two cohorts [5].
For respiratory isolates conversion rates in Acinetobacter spp., Pseudomonas spp., Enterobacter spp., E. coli or Klebsiella spp. during SDD were 0.7 per 1000 patient days. In that study 1 of 17 and 3 of 9 conversions in intestinal colonization, and 4 of 12 conversions in respiratory colonization (standard care, SOD and SDD combined) were in Enterobacter species [5].

WGS provided essential information for interpreting the molecular epidemiology of Enterobacter spp. in these ICUs. Plasmids do not belong to the core genome, and are not taken into account in the analysis of WGS. Plasmid-based colistin resistance genes ( $m c r-1$ to 4$)$ were not detected in any of the sequenced isolates (data not shown). The relatively low correlation between clustering and presence of ESBL may have resulted from horizontal transfer of plasmids encoding ESBLs. There is limited data on the number of expected SNPs in clonal transmission. In a set of presumed outbreak isolates based on identical PFGE patterns from two patients isolates had less than 22 SNPs, while isolates from a third patient were distinct with a distance greater than 150 SNPs [17]. It was concluded that the third patient was unlikely to have been associated with a transmission event involving one of the other two patients. We found two clusters with up to 126 SNPs.

\section{Conclusions}

A high incidence of carriage and infection with MREb isolates in patients admitted in the ICU in a Dutch hospital was noted in June 2014. It was hypothesized that the use of SDD in this ICU since 1990 had contributed to this high incidence, either due to repeated events of endogenous selection or due to incidental introduction 
of MREb strains followed by clonal transmission. These data demonstrate a persistent low prevalence of carriage with MREb, most probably maintained by incidental cross-transmission events and repeated (sporadic) introductions of carriers. These findings are compatible with a scenario in which SDD caused repeated events of endogenous selection, but in which existing infection control measures prevented uncontrolled spread, as well as with a scenario in which SDD prevented higher frequencies of carriage and subsequent uncontrolled spread due to insufficient infection control measures. Our findings also demonstrate that SDD must be accompanied by appropriate microbiological surveillance and good adherence to infection control measures in the unit.

\section{Additional file}

Additional file 1: Table S1. Colistin minimum inhibitory concentrations (MIC) as determined by Vitek and Etest for 100 Enterobacter isolates. (DOCX $15 \mathrm{~kb})$

\section{Abbreviations}

CSF: Cerebrospinal fluid; ESBL: Extended-spectrum beta-lactamase; ICU: Intensive care unit; MIC: Minimum inhibitory concentration; MREb: Multiresistant Enterobacter cloacae; NDM: New Delhi metallo-beta-lactamase; PFGE: Pulsed-field gel electrophoresis; SDD: Selective decontamination of the digestive tract; SNP: Single mucleotide polymorphism; SOD: Selective oropharyngeal decontamination; WGS: Whole genome sequencing

\section{Funding}

This study was supported by internal funding.

\section{Availability of data and materials}

The datasets used and/or analysed during the current study are available from the corresponding author on reasonable request.

\section{Authors' contributions}

$M D, M L v H, A M, L G, C J, E L, T R, R S, A F$ and $M B$ contributed to conception and design of the article. MD collected and analysed data. AM and CJ were responsible for microbiological tests. JS performed WGS experiments. JR and AF analysed WGS data. MD, JR, AF and MB wrote the first version of the manuscript. All authors critically revised the manuscript, and read and approved the final manuscript.

Preliminary data was presented at the 26th European Congress of Clinical Microbiology and Infectious Diseases (O120), and a preliminary version was published as part of the PhD thesis "Epidemiology of multidrug-resistant Enterobacteriaceae" (M.J.D. Dautzenberg, ISBN/EAN 978-90-393-6419-2).

Ethics approval and consent to participate

Not applicable.

\section{Competing interests}

The authors declare that they have no competing interests.

\section{Publisher's Note}

Springer Nature remains neutral with regard to jurisdictional claims in published maps and institutional affiliations.

\section{Author details}

'Department of Medical Microbiology, University Medical Center Utrecht, Utrecht, the Netherlands. ${ }^{2} J u l i u s$ Center for Health Sciences and Primary Care, University Medical Center Utrecht, Utrecht, the Netherlands. ${ }^{3}$ Department of Medical Microbiology, Radboud University Medical Center, Nijmegen, the Netherlands. ${ }^{4}$ Department of Medical Microbiology, Haaglanden Medisch
Centrum, The Hague, the Netherlands. ${ }^{5}$ Department of Medical Microbiology, Alrijne Hospital, Leiden, the Netherlands. ${ }^{6}$ Department of Internal Medicine, Haaglanden Medisch Centrum, The Hague, the Netherlands. ${ }^{7}$ Department of Intensive Care Medicine, Haaglanden Medisch Centrum, The Haque, the Netherlands.

Received: 18 March 2018 Accepted: 1 May 2018

Published online: 22 May 2018

\section{References}

1. Mezzatesta ML, Gona F, Stefani S. Enterobacter cloacae complex: clinical impact and emerging antibiotic resistance. Future Microbiol. 2012;7:887-902.

2. Kocsis B. The investigation of colistin resistance mechanisme in clinical isolates of Enterobacter asburiae. At: 25th European congress of clinical microbiology and Infectious Diseases; 2015. p. P1007.

3. Kluytmans J. Plasmid-encoded colistin resistance: mcr-one, two, three and counting. Euro Surveill. 2017;22(31):pii=30588.

4. de Smet AMGA, Kluytmans JAJW, Cooper BS, et al. Decontamination of the digestive tract and oropharynx in ICU patients. N Engl J Med. 2009:360:20-31.

5. Oostdijk EAN, Smits L, De Smet AMG, Leverstein-Van Hall MA, Kesecioglu J, Bonten MJM. Colistin resistance in gram-negative bacteria during prophylactic topical colistin use in intensive care units. Intensive Care Med. 2013;39:653-60

6. Halaby T, Al Naiemi N, Kluytmans J, van der Palen J, Vandenbroucke-Grauls CMJE. Emergence of colistin resistance in Enterobacteriaceae after the introduction of selective digestive tract decontamination in an intensive care unit. Antimicrob Agents Chemother. 2013;57:3224-9.

7. European Committee on Antimicrobial Susceptibility Testing. Clinical breakpoints - bacteria v3.1. http://www.eucast.org/fileadmin/src/media/ PDFs/EUCAST_files/Breakpoint_tables/Breakpoint_table_v_3.1.pdf. Accessed 18 May 2018

8. Toolkit for processing sequences in FASTA/Q formats. https://github.com/ Ih3/seqtk. Accessed 18 May 2018.

9. Bankevich A, Nurk S, Antipov D, et al. SPAdes: a new genome assembly algorithm and its applications to single-cell sequencing. J Comput Biol. 2012;19:455-77.

10. Seemann T. Prokka: rapid prokaryotic genome annotation. Bioinformatics. 2014;30:2068-9.

11. Camacho C, Coulouris G, Avagyan V, et al. BLAST+: architecture and applications. BMC Bioinf. 2009;10:421.

12. Ekseth OK, Kuiper M, Mironov V. orthAgogue: an agile tool for the rapid prediction of orthology relations. Bioinformatics. 2014;30:734-6.

13. van Dongen $S$, Abreu-Goodger C. Using MCL to extract clusters from networks. Methods Mol Biol. 2012:804:281-95.

14. Croucher NJ, Page AJ, Connor TR, et al. Rapid phylogenetic analysis of large samples of recombinant bacterial whole genome sequences using Gubbins. Nucleic Acids Res. 2015;43:e15.

15. Price MN, Dehal PS, Arkin AP. FastTree 2-approximately maximumlikelihood trees for large alignments. PLoS One. 2010;5:e9490.

16. Matuschek E, Åhman J, Webster C, Kahlmeter G. Evaluation of five commercial MIC methods for colistin antimicrobial susceptibility testing for gram-negative bacteria, at: 27th European congress of clinical microbiology and Infectious Diseases; 2017. p. P0161.

17. Reuter S, Ellington MJ, Cartwright EJP, et al. Rapid bacterial whole-genome sequencing to enhance diagnostic and public health microbiology. JAMA Intern Med. 2013;173:1397-404.

\section{Ready to submit your research? Choose BMC and benefit from:}

- fast, convenient online submission

- thorough peer review by experienced researchers in your field

- rapid publication on acceptance

- support for research data, including large and complex data types

- gold Open Access which fosters wider collaboration and increased citations

- maximum visibility for your research: over $100 \mathrm{M}$ website views per year

At BMC, research is always in progress.

Learn more biomedcentral.com/submissions 